From the Medical University of Vienna, Vienna, Austria; Wake Forest University School of Medicine, Winston-Salem; GlaxoSmithKline, Durham, NC: Washington University School of Medicine, St Louis, MO; and Bostwick Laboratories, Glen Allen, VA.

Submitted August 19, 2010; accepted January 14, 2011; published online ahead of print at www.jco.org on March 28, 2011

Supported by GlaxoSmithKline. G.L.A received research funding from the National Cancer Institute and National Institute of Diabetes and Digestive and Kidney Diseases.

Authors' disclosures of potential conflicts of interest and author contributions are found at the end of this article.

Clinical Trials repository link available on JCO.org.

Corresponding author: Michael Marberger, MD, FRCS (ed), Department of Urology, Medical University of Vienna, Waehringer Guertel 18-20, A-1090, Vienna, Austria; e-mail: Michael Marberger@A1.net.

(C) 2011 by American Society of Clinical Oncology

0732-183X/11/2913-1744/\$20.00 DOI: 10.1200/JCO.2010.32.1646

\title{
Biopsy Misidentification Identified by DNA Profiling in a Large Multicenter Trial
}

Michael Marberger, John D. McConnell, Ivy Fowler, Gerald L. Andriole, David G. Bostwick, Matthew C. Somerville, and Roger S. Rittmaster

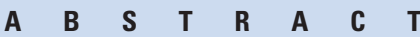

\section{Purpose}

The Reduction by Dutasteride of Prostate Cancer Events (REDUCE) prostate cancer risk reduction study randomly assigned 8,231 men to dutasteride or placebo for 4 years. Protocol-mandated biopsies were obtained after 2 and 4 years. After the discovery of three cases of biopsy sample misidentification in the first 2 years, all protocol-mandated biopsy samples were DNA tested to verify biopsy identity.

\section{Methods}

Biopsy and blood DNA profiling was performed retrospectively for the year 2 scheduled biopsies and prospectively for the year 4 scheduled biopsies. Toward the end of year 2, multiple changes were made to improve sample handling and chain of custody.

\section{Results}

Of the 6,458 year 2 and 4,777 year 4 biopsies, 26 biopsies reflecting 13 sample handling errors at year $2(0.4 \%)$ and one biopsy reflecting one sample handling error at year $4(0.02 \%)$ were confirmed to be mismatched to the patient for whom they were originally submitted. Of 6,733 reference blood samples profiled, $31(0.5 \%)$ were found to be mismatched to the patient's verified identity profile. Sample identification errors occurred at local research sites and central laboratories.

\section{Conclusion}

Biopsy misidentification is a potential problem in clinical laboratories and clinical trials. Until now, biopsy misidentification has not been studied in the setting of a large, multinational clinical trial. In the REDUCE study, process improvement initiatives halfway through the trial dramatically reduced biopsy mismatches. The potential for biopsy mismatches in clinical trials and clinical practice is an under-recognized problem that requires rigorous attention to details of chain of custody and consideration of more widespread DNA identity testing.

\section{J Clin Oncol 29:1744-1749. (C) 2011 by American Society of Clinical Oncology}

\section{INTRODUCTION}

Tissue samples taken for pathologic analysis can be inadvertently mislabeled between the time a sample is taken and the time of pathology review. ${ }^{1}$ In the case of biopsy samples, such mislabeling of patients' samples can have serious consequences for patient management because treatment decisions are most frequently driven by pathologic findings on biopsy.

In the Reduction by Dutasteride of Prostate Cancer Events (REDUCE) trial of dutasteride for prostate cancer risk reduction, near the end of the year 2 study biopsy period, two positive biopsy samples were found not to correlate with subsequent surgical pathologic findings, and DNA identity testing confirmed that the biopsy samples did not belong to the patient identified as the owner.
Voluntary DNA identity testing revealed a third biopsy mismatch, leading to mandatory testing of all study-mandated biopsies retrospectively and prospectively. We now report the results of the DNA identity testing, including the effectiveness of procedures instituted to address the problem.

\section{METHODS}

\section{The REDUCE Study Design}

REDUCE was a double-blind, placebo-controlled study (ClinicalTrials.gov identification No. NCT00056407) sponsored by GlaxoSmithKline (Durham, NC) with more than 800 sites in 42 countries (Figure 1). It was designed to determine whether dutasteride $0.5 \mathrm{mg}$ daily decreases the risk of biopsy-detectable prostate cancer compared with placebo over 4 years in a population at increased risk of developing the disease. Patients were recruited between 


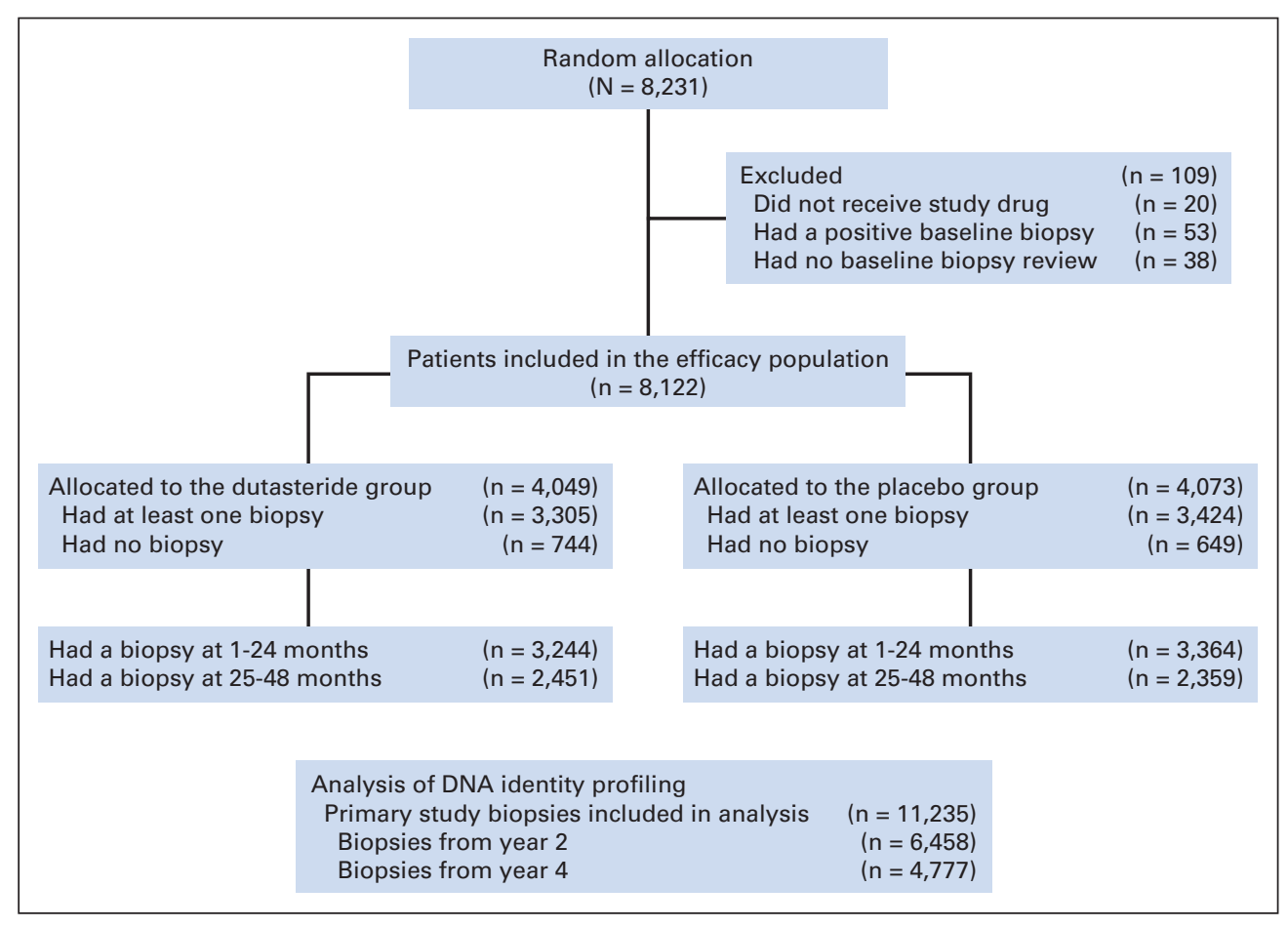

Fig 1. CONSORT diagram.

March 2003 and December 2004. The rationale and study design for the REDUCE study have been published. ${ }^{2}$ The protocol was approved by the relevant institutional review board of each study site, and all patients gave written informed consent. All relevant institutional review boards were notified of the DNA biopsy identity testing, and any institutional review board with initial concerns provided agreement after explanation of the implications for patient safety and the limited nature of the DNA testing. A total of 8,231 men underwent random assignment. All patients had a single negative baseline prostate needle biopsy with six to 12 core samples, and repeat 10 -core biopsies were performed after 2 and 4 years. ${ }^{2}$ All protocol-mandated biopsies were processed at a central pathology laboratory (Clinical Laboratory Improvement Amendments/College of American Pathologists certified) based in the United States and read by the central pathologist. Protocol-independent, for-cause biopsies were permitted as deemed necessary by the clinician. Protocolindependent biopsies were processed by local pathology laboratories and excluded from DNA identity testing. Serum and plasma biomarker samples were collected from all patients every 6 months and stored for future research according to study protocol, and a blood sample for DNA extraction was collected in patients who consented to genetic testing. DNA for identity testing was obtained from a portion of either of these samples.

\section{Biopsy Handling}

All study site personnel involved in handling REDUCE study biopsies were required to undergo training in both video and handbook formats, which covered specifics of the standardized study biopsy acquisition, chain of custody, and biopsy packaging and shipping to the central pathology laboratory. Laboratory personnel training was the responsibility of each laboratory and specific to the study processes as outlined in the study protocol. Training for study site personnel was the responsibility of the GlaxoSmithKline study team and clinical research monitors, with guidance protocols provided by the central study management team. After discovery of the first few mismatches, sites were retrained with emphasis on maintaining the biopsy chain of custody.

A set of two study biopsy requisitions was completed at every study biopsy. The patient number was written on the requisitions and the requisition labels, and each formalin vial was labeled. The scheduled study biopsy cores were placed in these vials. Vials were placed in the biopsy box, and a requisition label was placed on the exterior of the sealed box. This box was placed inside a plastic biohazard bag, which was then sealed.
Associated biopsy requisitions were placed in the outer pocket of the bag. Biopsies were shipped by the site to a central blood laboratory in the United States or United Kingdom for intake and tracking. The central blood laboratory entered identifying information into its system and replaced the requisition in the pocket of the biohazard bag, without opening the sample box. The biopsy samples received daily were then forwarded in bulk to the central pathology laboratory for processing and review.

\section{DNA Identity Testing}

At the end of the second year, after the first three biopsy mismatches were discovered, a mandatory biopsy identity testing program was instituted. Year 2 biopsies were evaluated retrospectively, and year 4 biopsies were evaluated prospectively, immediately after pathologic interpretation. DNA extraction from paraffin-embedded tissue sections and DNA identity testing were performed at the Laboratory Corporation of America Holdings Forensic Identity Laboratory (Durham, NC). DNA extraction was performed either manually with organic chemicals or using the Invitrogen iPrep Purification Instrument with ChargeSwitch chemistry (Invitrogen, Carlsbad, CA). Up to 16 short tandem repeat loci on DNA were analyzed using the laboratory's standard procedures. ${ }^{3}$ Identity was verified by comparing the DNA profile of submitted prostate biopsy tissue with the patient's stored blood sample. For all patients who underwent protocol-mandated 2- and 4-year biopsies, a minimum of two negative slides and/or two negative blocks from each biopsy were used for identity testing.

In instances where the biopsy sample and blood reference profiles did not match, further aliquots of stored blood samples were tested until the source of the sample misidentification could be confirmed as either the biopsy tissue or one or more of the reference blood samples. A verified identity profile was confirmed when the blood and biopsy samples matched or, in the case of a mismatch between the blood and biopsy samples, when two different blood samples from the same patient matched. Profiles of misidentified biopsy samples were then tested against those of other REDUCE study patients until a crossmatch for the switched sample was identified in another REDUCE patient or the biopsy-switched sample was determined to originate from a nonREDUCE patient. Notification of mismatch and revised biopsy results were provided verbally to the investigator immediately, followed by an amended pathology report sent to the investigative site. Investigators informed patients 


\begin{tabular}{|ccc|}
\hline \multicolumn{2}{c|}{ Table 1. DNA Identity Profiling Results for Year 2 and 4 Study Biopsies } \\
\hline \multicolumn{2}{c}{ Time Period and } & No. of \\
DNA Match Status & Samples & $\%$ \\
\hline Years 1 and 2 & & \\
Total biopsy samples & 6,458 & \\
DNA analysis and match & 6,429 & 99.55 \\
DNA analysis and mismatch & 26 & 0.4 \\
No DNA analysis & 3 & 0.05 \\
Years 3 and 4 & 4,777 & \\
Total biopsy samples & 4,775 & \\
DNA analysis and match & 1 & 0.96 \\
DNA analysis and mismatch & 1 & 0.02 \\
No DNA analysis & & \\
\hline
\end{tabular}

and discussed further clinical decisions, as needed. Crossmatching of misidentified blood samples was not pursued in patients other than those enrolled onto the REDUCE trial.

\section{Sample Handling and Process Improvements}

Before any year 4 biopsy, educational efforts and process improvements were instituted based on the recommendations of external pathology process improvement consultants from Roche Healthcare Services Division (Indianapolis, IN). REDUCE trial personnel worked with investigative sites to review the importance of specimen labeling, separation of patients undergoing biopsy, and physical separation of specimens during preparation and packaging.
Regional meetings were held with local study personnel and monitors, focusing on biopsy specimen chain of custody.

At the central blood laboratory, process improvement efforts included retraining of laboratory personnel to minimize handling of the pathology specimens. Improvements were made in written communication to the central pathology laboratory when potential identity issues were noted during receipt and tracking.

At the central pathology laboratory, changes to a specimen's critical identifiers in the data systems (eg, patient number, date of birth, site number) were restricted to quality control personnel. REDUCE study tissue cassettes were color coded to easily distinguish these from other samples present in the laboratory. The physical space used for sample registration, accessioning, histology, and slide boarding was improved to minimize work overlap and handling errors. Later in the study, bar coding was added to all REDUCE samples on receipt at the central pathology laboratory to reduce potential human error in reading and tracking patient numbers on samples or documents once a specimen was received. Finally, retrospectively for mismatched samples in year 2 and prospectively for year 4 biopsies, visual inspection of slides and blocks was added to ensure slides for each patient matched and were paired with the blocks from which they were derived.

\section{RESULTS}

\section{Year 2 and 4 Scheduled Biopsies}

For the efficacy population (randomly assigned patients with negative baseline biopsy who received $\geq$ one dose of study medication $),{ }^{4}$ a total of 11,235 primary study biopsy samples (6,458 samples

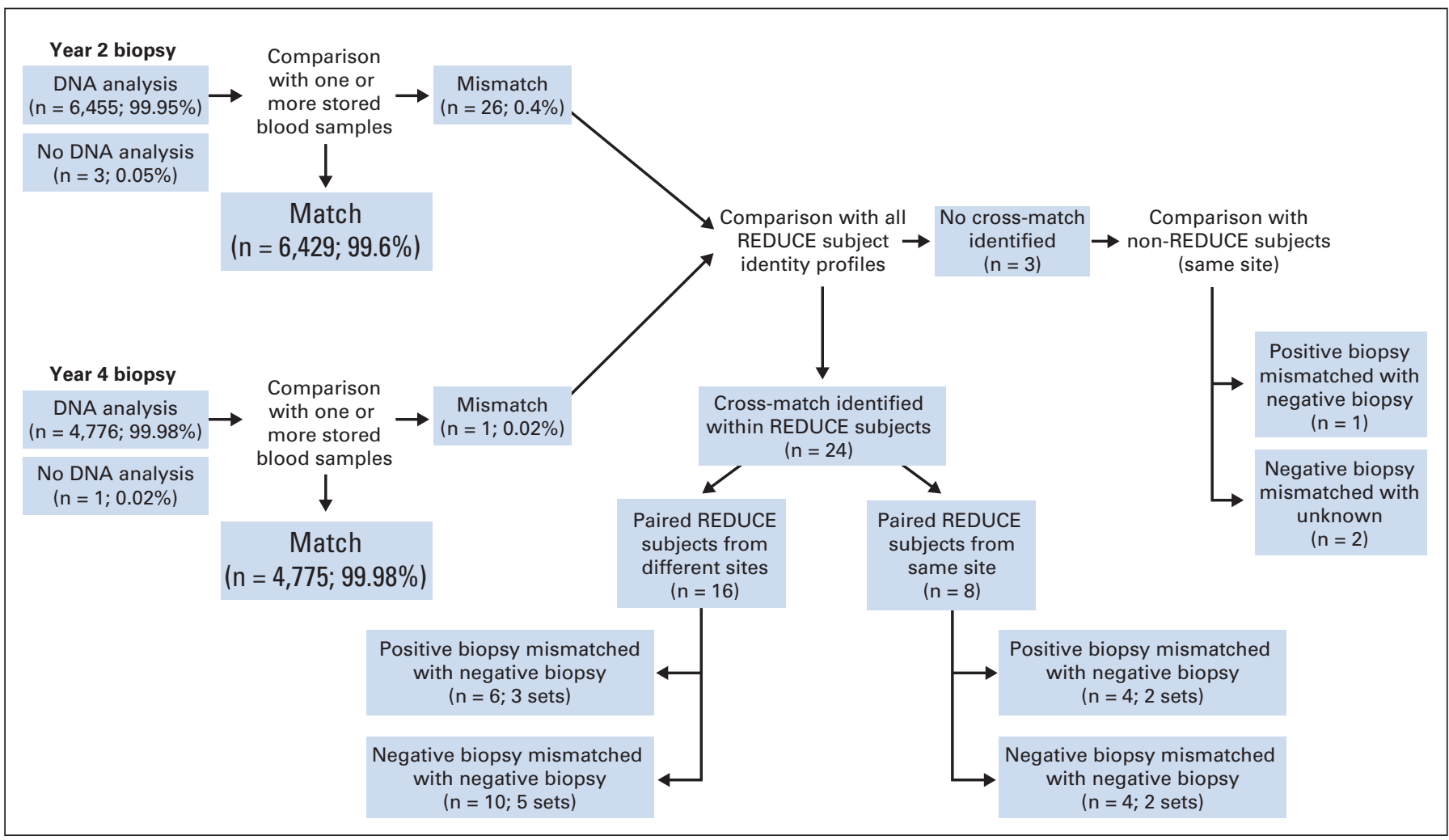

Fig 2. Biopsy identification testing and types of mismatches. Biopsy mismatches were identified in 26 year-2 biopsies and one year-4 biopsy. For biopsy

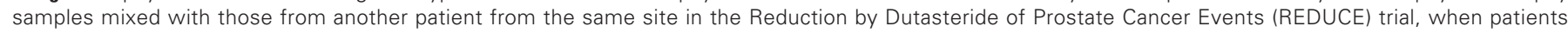

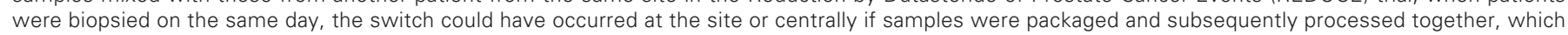

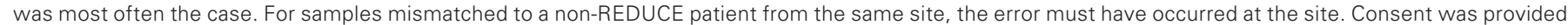
by non-REDUCE patients for any DNA testing. 
at year 2 and 4,777 samples at year 4) were DNA identity tested (Table 1). Crossmatched cases were identified for all biopsy mismatch cases, and results were corrected through an amended pathology report to the site and correction to the study data. Of all biopsies tested, 26 year 2 biopsies and one year 4 biopsy were confirmed to be mismatched to the patient for whom they were originally submitted. Figure 2 shows matched and mismatched biopsies and the types of mismatches that occurred. The 27 affected REDUCE patients represent 14 instances of biopsy switches, or 14 instances of human error (12 instances involved a switch within REDUCE patient biopsies; one instance involved a three-way switch between two REDUCE patients and a non-REDUCE patient; and one instance involved a switch between a REDUCE patient and a non-REDUCE patient). In most cases, the specific source of the error could not be determined. If specimen vials were labeled correctly by the site, errors involving handling the specimen requisitions should not have resulted in biopsy mismatches independently because subsequent steps in the central pathology process should have detected a mismatch between the requisitions and the vials sealed within the biopsy box.

\section{DNA Match Results: Reference Blood Samples}

During the biopsy matching process, 6,733 reference blood samples were tested to ensure a verified identity reference profile for all patients who had a study-required biopsy. Only one patient who had a primary biopsy had no reference blood profile because he ordered destruction of his samples on withdrawal from the study. From the 6,733 blood samples profiled, 31 blood samples were found to be mismatched to the patient's verified identity profile (Table 2).

\section{Challenges to Sample Identity Profiling}

DNA profiling presented several difficulties. For four of the biopsies tested (three year 2 biopsies and one year 4 biopsy), DNA matching was not possible because there was either no paired stored blood sample available or one part of the paired sample used for matching did not yield enough DNA for adequate identity profiling. In some cases, sample contamination or poor DNA yield made further steps at DNA isolation and testing necessary to obtain an adequate identity profile. For the 11,235 biopsy cases submitted for profiling (two slides per biopsy case), $13.3 \%$ were found to be contaminated with other sources of DNA, presumably during processing or shipment. Of the 11,235 cases, 171 biopsy cases $(1.5 \%)$ had enough contamination on both submitted slides to necessitate requests for more slide tissue or use of tissue blocks for further testing before adequate profiling could be obtained. Poor DNA yield from biopsy slides in 305 biopsy cases $(2.7 \%)$ led to the need for testing of further biopsy slides or available tissue blocks to obtain a profile. The amount of biopsy tissue needed to obtain a DNA profile determined the amount of tissue used for DNA testing. In most cases, the two slides provided yielded sufficient DNA

\begin{tabular}{|ccr|}
\hline \multicolumn{3}{|c|}{ Table 2. DNA Identity Profiling Results in Blood Reference Samples } \\
\hline & No. of & \\
\hline DNA Match Results & Samples & \\
\hline Total blood samples tested & 6,733 & \\
Blood analysis and match & 6,702 & 99.5 \\
Blood analysis and mismatch & 31 & 0.5 \\
\hline & & \\
\hline
\end{tabular}

for testing; however, in some cases, additional tissue from slides or tissue blocks was requested to complete DNA profiling, as described earlier.

Although serum and plasma protein biomarker blood samples are not prepared for or expected to contain large amounts of DNA, in most cases, DNA isolation from those samples was successful. In some cases, either because of sample contamination or poor DNA yield, a second sample was required. As expected, for patients who had a stored DNA sample for reference, problems with profiling were rare. Of the 6,733 unique blood samples tested, 3,012 were stored as either serum or plasma protein biomarker samples, and 3,721 were stored DNA samples from the study. Of the 3,012 serum/plasma biomarker samples tested, $53(1.8 \%)$ failed to provide an adequate profile on testing-46 $(1.5 \%)$ as a result of inadequate DNA yield and seven $(0.2 \%)$ as a result of contamination of the blood sample with DNA from another individual. Of the 3,721 reference DNA blood samples tested for identity profiling, $23(0.6 \%)$ failed to provide an adequate profile on testing-18 $(0.5 \%)$ as a result of inadequate DNA yield and five $(0.1 \%)$ as a result of contamination of the sample from another individual.

\section{DISCUSSION}

To our knowledge, REDUCE is the first published study to examine prostate biopsy misidentification rates in a large international population using a central pathology laboratory. Large, complex, multinational studies present logistical challenges that may require prospective planning and laboratory inspections to ensure consistent chain of custody and sample processing. To date, the magnitude of the problem of identity mismatch of pathology specimens is unknown. Few studies have been published that document the use of DNA profiling to identify prostate biopsy identity errors. ${ }^{1,5-8}$ In most of these studies, DNA profiling was only used for patients in whom cancer could not be identified within the prostatectomy surgical specimen (pT0 patients) despite testing positive by needle biopsy. Moreover, the number of patients for whom DNA identity profiling was performed in these studies was small, ranging from two to six patients. ${ }^{1,7,8}$ The largest study analyzed included the identity profile of 46 patients, in which only one mismatch was found. ${ }^{6}$ This historically small sampling of DNA profiling to identify sample mismatch rates makes it impossible to quantify the extent of the pathology sample mismatches in clinical practice. Many cases of prostate biopsy misidentification are likely to go undetected because many patients do not choose surgical treatment, in which case the possibility of an incorrect pathologic diagnosis never arises. The challenge of identifying biopsy mismatches is further confounded because men with an erroneously positive prostate biopsy may, in fact, have prostate cancer at surgery, despite the true biopsy being negative. In the REDUCE trial, only two of the 14 pairs of sample switches during the retrospective evaluation of 2-year biopsies were suspected based on evaluation of surgical tissue. One was suspected because of a negative radical prostatectomy after a positive biopsy, and the other was suspected when the grade and distribution of cancer on radical prostatectomy did not match the grade and distribution on biopsy.

Several aspects of the REDUCE trial, and research trials in general, may have increased the likelihood of biopsy misidentification. 
These aspects included the following: patient numbers replaced patient names; research charts looked similar; the same number of biopsy cores was obtained for each patient; and often, more than one REDUCE patient was biopsied at a study site, with samples batched and shipped to central pathology on the same day and time. Whereas a typical pathology laboratory sees many different types of biopsies one after the other, for the REDUCE study, all samples were reviewed at the same central laboratory and often in batches of like biopsies. Although it was often impossible to pinpoint the exact location of a switch in biopsy samples, evidence indicates that errors occurred throughout the chain of custody, from the investigative site to the central pathology laboratory. As such, it is not unreasonable to suspect that similar errors can occur in routine clinical practice, the frequency of which is unknown.

Despite the multiple sources of possible error for an individual case, the rigorous process improvement effort that took place halfway through the study clearly demonstrated the effectiveness of heightened awareness, attention to detail, and chain of custody and spatial changes to minimize the chances that two samples could be switched. This led to a reduction in biopsy handling errors from 13 instances in the first 2 years of the study to one instance in the second 2 years.

Although DNA identification was successful on nearly all biopsies tested, some limitations exist. The analysis assumes that all biopsy cores in a sample came from the same patient. A partial biopsy sample switch could have been missed. Such a switch could be detected by visual inspection by the pathologist to ensure that the slides matched the corresponding blocks for the patient. Errors could also occur in the reporting of pathology results, which would not be detected through identity profiling of the sample. In addition, although the success rate for obtaining profiles from biopsy tissue not originally prepared for profiling was high, a few samples did not provide enough DNA to adequately confirm identity.

Should DNA identity testing become routine in clinical trials with pathology end points? Sample misidentification probably would not affect the scientific accuracy of studies of this size; however, identity testing certainly is a consideration for patient welfare if important clinical decisions are based on test results. At a cost of as much as US $\$ 1,500$ per test for case-by-case testing, cost is a limiting factor. Whether or not such identity testing is performed, all aspects of sample handling, from obtaining tissue to reporting the results, should be carefully examined before the start of a clinical trial and perhaps routinely in clinical practice (Table 3). For example, methods such as bar coding can be instituted to minimize mistakes in reading or transcribing identification codes, to provide audit tracking of specimens to highlight areas of sample cross over during processing, and to highlight areas of processing that need better quality control. Although this study does not provide information on the prevalence of mismatches in routine clinical practice, the biopsying physician and the pathology laboratory should recognize the possibility for human error and use robust quality control processes to substantially reduce, if not prevent, errors. Nevertheless, in the end, it is attention to detail at every step of the process that is needed to minimize human errors that are fundamental to each case of sample misidentification.

In conclusion, during retrospective DNA identity testing of biopsy samples during the first 2 years of the REDUCE trial, $0.4 \%$ of biopsies $(n=26)$ were found to be mismatched. This was reduced to a single mismatch during the second 2 years, after institution of a rigorous review of sample handling procedures. The potential for
Table 3. Key Strategies to Address Biopsy Identification Errors in Clinical Trials

Strategies

1. Conduct prestudy review of sample handling and pathology processes by independent expert.

2. Ensure all study site and laboratory personnel are trained on standard operating procedures designed to avoid sample identity errors.

3. Patient identifier should be formatted as a combination of letters and numbers, rather than numbers alone.

4. Avoid scheduling two study patients for biopsy back to back or having supplies for two patients in the same room at the same time.

5. Label pathology kits and requisitions at the time of the biopsy procedure, not in advance.

6. Have the patient verify accuracy of identifying information on his/her biopsy.

7. Store at least two blood samples (or prepared mouth swabs) at different times for each study participant, in anticipation of use for identity testing.

8. Store an extra set of unstained covered slides and, in case slides are contaminated by exogenous DNA, two biopsy blocks.

9. Consider DNA identity testing on all samples concurrent with, or immediately after, pathologic evaluation.

sample misidentification should be a key concern for any large clinical study involving pathologic samples, as well as for routine clinical practice. In light of this concern and the success achieved in this study in reducing sample identity mismatches, we recommend that DNA testing of patient samples be a mandatory feature for large multinational clinical studies where testing of biologic samples via a central laboratory is required as part of the main study end points.

\section{AUTHORS' DISCLOSURES OF POTENTIAL CONFLICTS OF INTEREST}

Although all authors completed the disclosure declaration, the following author(s) indicated a financial or other interest that is relevant to the subject matter under consideration in this article. Certain relationships marked with a " $U$ " are those for which no compensation was received; those relationships marked with a " $C$ " were compensated. For a detailed description of the disclosure categories, or for more information about ASCO's conflict of interest policy, please refer to the Author Disclosure Declaration and the Disclosures of Potential Conflicts of Interest section in Information for Contributors.

Employment or Leadership Position: Ivy Fowler, GlaxoSmithKline (C); David G. Bostwick, Bostwick Laboratories (C); Matthew C. Somerville, GlaxoSmithKline (C); Roger S. Rittmaster, GlaxoSmithKline (C) Consultant or Advisory Role: Michael Marberger, GlaxoSmithKline (C); John D. McConnell, GlaxoSmithKline (C); Gerald L. Andriole, Amgen (C), Augmenix (C), Caris (C), EMD Serono (C), France Foundation (C), GenProbe (C), GlaxoSmithKline (C), Myriad Genetics (C), Nema Steba (C), Onconome (C), Veridex (C), Bayer (C), Cambridge Endo (C), Ortho-Clinical Diagnostics (C) Stock Ownership: Ivy Fowler, GlaxoSmithKline; Gerald L. Andriole, Envisioneering, Viking Medical; David G. Bostwick, Bostwick Laboratories, GlaxoSmithKline; Matthew C. Somerville, GlaxoSmithKline; Roger S. Rittmaster, GlaxoSmithKline Honoraria: Michael Marberger, GlaxoSmithKline, Merck Sharp \& Dohme; Gerald L. Andriole, GlaxoSmithKline Research Funding: Gerald L. Andriole, Aeterna Zentaris; David G. Bostwick, GlaxoSmithKline, Bioniche, EDAP, Spectrum Pharmaceuticals, GTx, Accuray, Stiefel, sanofi-aventis, Capstone, Nippon Kayaku, Daiichi Sankyo/Covance, Bostwick Laboratories Expert Testimony: Michael Marberger, GlaxoSmithKline (C); Roger S. Rittmaster, GlaxoSmithKline (C) Other Remuneration: None 


\section{AUTHOR CONTRIBUTIONS}

Conception and design: Michael Marberger, John D. McConnell, Ivy Fowler, Gerald L. Andriole, Roger S. Rittmaster Administrative support: Ivy Fowler, David G. Bostwick
Provision of study materials or patients: Ivy Fowler, David G. Bostwick Collection and assembly of data: Michael Marberger, Ivy Fowler, Matthew C. Somerville, Roger S. Rittmaster Data analysis and interpretation: All authors Manuscript writing: All authors

Final approval of manuscript: All authors

\section{REFERENCES}

1. Goldstein NS, Bégin LR, Grody WW, et al: Minimal or no cancer in radical prostatectomy specimens: Report of 13 cases of the "vanishing cancer phenomenon.” Am J Surg Pathol 19:1002-1009, 1995

2. Andriole G, Bostwick D, Brawley O, et al: Chemoprevention of prostate cancer in men at high risk: Rationale and design of the Reduction by Dutasteride of Prostate Cancer Events (REDUCE) trial. J Urol 172:1314-1317, 2004
3. Kimpton CP, Gill P, Walton A, et al: Automated DNA profiling employing multiplex amplification of short tandem repeat loci. PCR Methods Appl 3:13-22, 1993

4. Andriole GL, Bostwick DG, Brawley OW, et al: Effect of dutasteride on the risk of prostate cancer. N Engl J Med 362:1192-1202, 2010

5. DiGiuseppe JA, Sauvageot J, Epstein JI: Increasing incidence of minimal residual cancer in radical prostatectomy specimens. Am J Surg Pathol 21:174-178, 1997

6. Cao D, Hafez M, Berg K, et al: Little or no residual prostate cancer at radical prostatectomy:
Vanishing cancer or switched specimen? A microsatellite analysis of specimen identity. Am J Surg Pathol 29:467-473, 2005

7. Trpkov K, Gao Y, Hay R, et al: No residual cancer on radical prostatectomy after positive 10core biopsy: Incidence, biopsy findings, and DNA specimen identity analysis. Arch Pathol Lab Med 130:811-816, 2006

8. Mazzucchelli R, Barbisan F, Tagliabracci A, et al: Search for residual prostate cancer on $\mathrm{pTO}$ radical prostatectomy after positive biopsy. Virchows Arch 450:371-378, 2007

\section{EVERY 6 MINUTES Research Published in JCO Is Cited in Other Peer-Reviewed Journals}

As reported by Thomson Reuters in its 2008 Journal Citation Report ${ }^{\circledR}$, Journal of Clinical Oncology's Impact Factor has increased to 17.157 from 15.484. This is JCO's fourth straight yearon-year increase.

In number of citations, JCO ranks second among 141 oncology journals and ranks 25th among all 6,598 scientific journals surveyed. JCO articles were cited more than 97,000 times in 2008-a $20 \%$ increase over the previous year.

JCO has published so much research-changing and practice-changing science over the years that, in 2008, a JCO article was cited every 6 minutes, on average, in another peer-reviewed journal.

If you want to have your research read by the largest, most discerning international audience, you need to publish in $J C O$. And if you want to read the most important research in clinical oncology, you need to subscribe to JCO.

To submit a manuscript, visit submit.jco.org.

To subscribe or activate, visit jco.org/subscriptions. 